\title{
Article
}

\section{Bscl2 Deficiency Does Not Directly Impair the Innate Immune Response in a Murine Model of Generalized Lipodystrophy}

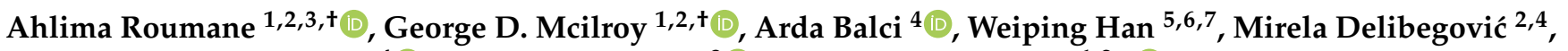 \\ Massimiliano Baldassarre ${ }^{4}(\mathbb{D})$, Philip Newsholme ${ }^{3}$ (D) and Justin J. Rochford ${ }^{1,2, *(D)}$ \\ 1 The Rowett Institute, University of Aberdeen, Foresterhill, Aberdeen AB25 2ZD, UK; \\ a.roumane.18@abdn.ac.uk (A.R.); g.mcilroy@abdn.ac.uk (G.D.M.) \\ 2 The Aberdeen Cardiovascular and Diabetes Centre, University of Aberdeen, Foresterhill, \\ Aberdeen AB25 2ZD, UK; m.delibegovic@abdn.ac.uk \\ 3 Curtin Health Innovation Research Institute, School of Pharmacy and Biomedical Sciences, Curtin University, \\ Perth, WA 6102, Australia; Philip.Newsholme@curtin.edu.au \\ 4 Institute of Medical Sciences, University of Aberdeen, Foresterhill, Aberdeen AB25 2ZD, UK; \\ r04ab17@abdn.ac.uk (A.B.); massimiliano.baldassarre@abdn.ac.uk (M.B.) \\ 5 Laboratory of Metabolic Medicine, Institute of Molecular and Cell Biology, Agency for Science, Technology \\ and Research (A*STAR), Singapore 138673, Singapore; wh10@cornell.edu \\ 6 Center for Neuro-Metabolism and Regeneration Research, Guangzhou Regenerative Medicine and Health \\ Guangdong Laboratory, Guangzhou 510700, China \\ 7 School of Laboratory Medicine and Life Sciences, Wenzhou Medical University, Wenzhou 325035, China \\ * Correspondence: j.rochford@abdn.ac.uk; Tel.: +44-(0)1224-437372 \\ + These authors contributed equally to this work.
}

Citation: Roumane, A.; Mcilroy, G.D.; Balci, A.; Han, W.; Delibegović, M.; Baldassarre, M.; Newsholme, P.; Rochford, J.J. Bscl2 Deficiency Does Not Directly Impair the Innate Immune Response in a Murine Model of Generalized Lipodystrophy. J. Clin. Med. 2021, 10, 441. https:// doi.org/10.3390/jcm10030441

Received: 7 December 2020

Accepted: 15 January 2021

Published: 23 January 2021

Publisher's Note: MDPI stays neutral with regard to jurisdictional claims in published maps and institutional affiliations.

\begin{abstract}
Congenital Generalized Lipodystrophy type 2 (CGL2) is the most severe form of lipodystrophy and is caused by mutations in the BSCL2 gene. Affected patients exhibit a near complete lack of adipose tissue and suffer severe metabolic disease. A recent study identified infection as a major cause of death in CGL2 patients, leading us to examine whether Bscl2 loss could directly affect the innate immune response. We generated a novel mouse model selectively lacking Bscl2 in the myeloid lineage (LysM-B2KO) and also examined the function of bone-marrow-derived macrophages (BMDM) isolated from global Bscl2 knockout (SKO) mice. LysM-B2KO mice failed to develop lipodystrophy and metabolic disease, providing a model to study the direct role of Bscl2 in myeloid lineage cells. Lipopolysaccharide-mediated stimulation of inflammatory cytokines was not impaired in LysM-B2KO mice or in BMDM isolated from either LysM-B2KO or SKO mice. Additionally, intracellular fate and clearance of bacteria in SKO BMDM challenged with Staphylococcus aureus was indistinguishable from that in BMDM isolated from littermate controls. Overall, our findings reveal that selective Bscl2 deficiency in macrophages does not critically impact the innate immune response to infection. Instead, an increased susceptibility to infection in CGL2 patients is likely to result from severe metabolic disease.
\end{abstract}

Keywords: BSCL2; seipin; congenital generalized lipodystrophy; immunity; macrophages

\section{Introduction}

Congenital Generalized Lipodystrophy (CGL) is a rare genetic disorder, where patients display a near complete lack of adipose tissue, resulting in insulin resistance, hepatic steatosis and hypertriglyceridemia [1]. The most severe form of CGL, CGL type 2 (CGL2), is caused by mutations affecting the protein seipin (encoded by BSCL2) [2]. Seipin is known to be a critical regulator of adipogenesis, as its loss prevents adipocyte differentiation in vitro [3,4] and adipose tissue development in vivo [5-8]. More recently, it has been revealed that seipin plays an important role as a scaffold protein, capable of binding numerous proteins that play critical functions in lipid droplet organization and triglyceride synthesis [9-12]. 
Rio Grande do Norte in Brazil has one of the highest rates of CGL2 prevalence. A recent study of CGL2 patients from this region revealed that this condition reduced lifespan by more than thirty years [13]. The authors found that one third of patients died as a result of liver disease, consistent with the severe metabolic dysfunction in these patients. Curiously, however, one third of patients died of infectious diseases. This led us to examine the role of seipin in the innate immune response, which has not previously been investigated and could contribute to the high incidence of deaths caused by infection.

Pathogen phagocytosis by macrophages and the progression and maturation of pathogen-containing phagosomes, a crucial event for the destruction of the pathogen, occurs in parallel with the formation of lipid droplets. Seipin has been shown to play important, evolutionarily conserved roles in lipid droplet biogenesis and dynamics in multiple cell types and species from yeast to man [14]. Within immune cells, lipid droplets synthesize and store inflammatory mediators and are considered structural markers of inflammation [15]. Interaction of lipid droplets with pathogen-containing phagosomes has been increasingly reported in response to infections and may contribute to destruction or contribute to the survival of the microorganism within host cells [16]. Thus, via altering the lipid droplet function, seipin could play an important role in the capacity of macrophages to respond appropriately to infections. To test this hypothesis, we ablated Bscl 2 specifically within the myeloid cell lineage of mice and characterized the innate immune response in this model and in bone-marrow-derived macrophages (BMDM) from global Bscl2 knockout (SKO) mice.

\section{Experimental Section}

\subsection{Animal Studies}

Myeloid-specific Bscl2 knockout (LysM-B2KO) mice were generated by crossing $B s c l 2^{(\mathrm{fl} / \mathrm{fl})}$ mice [8] with $B s c l 2^{(\mathrm{fl} / \mathrm{wt})}$ mice expressing Cre recombinase controlled by the Lyz2 promoter. Bscl2 knockout (SKO) mice were generated using a previously described method [17]. Briefly, fertilized Bscl2 ${ }^{\text {(fl/wt) }}$ one-cell embryos were incubated ex vivo with TAT-Cre recombinase (\#SCR508, Sigma, Gillingham, UK), then reimplanted into surrogate female dams. The resulting pups were screened by PCR to detect the deletion, and experimental colonies of SKO mice were then generated. All animal procedures were approved by the University of Aberdeen Ethics Review Board and performed under project license P94B395E0, approved by the UK Home Office under the Animals Scientific Procedures Act 1986. Unless stated otherwise, mice had ad libitum access to water and a standard chow $\operatorname{diet}($ CRM (P) 801722, Special Diets Services).

\subsection{Metabolic Studies}

Fat and lean mass were measured using the EchoMRITM-500 body composition analyzer (Zinsser Analytic GmbH, Eschborn, Germany). Mice were injected with $1 \mathrm{mg} / \mathrm{Kg}$ of lipopolysaccharides (LPS, Sigma) by intraperitoneal injection. For glucose tolerance tests, 5-h fasted mice received intraperitoneal injections of $2 \mathrm{mg} / \mathrm{g}$ D-glucose (Sigma). Blood glucose was monitored between 0 and 120 min by glucometer readings (AlphaTrak ${ }^{\circledR}$ II, Zoetisus, Parsippany-Troy Hills, NJ, USA) from tail punctures. Body temperatures were measured using a lubricated rectal probe inserted in mice maintained at standard housing temperatures both prior to and $3 \mathrm{~h}$ after LPS injections. Serum glucose levels were determined using the Glucose Colorimetric Assay Kit (Cayman Chemical, Ann Arbor, MI, USA); The insulin, Tnfa and Il-10 analysis was performed at the Core Biochemical Assay Laboratory (Cambridge, UK). The quantitative insulin sensitivity check index (QUICKI) was calculated as previously described [18]. QUICKI $=1 /[\log (\mathrm{I} 0)+\log (\mathrm{G} 0)]$, where I0 is fasting insulin $(\mu \mathrm{U} / \mathrm{mL})$ and $\mathrm{G} 0$ is fasting glucose $(\mathrm{mg} / \mathrm{dL})$. QUICKI is a dimensionless index without units. 


\subsection{Gene Expression}

RNA was extracted from tissues or cells using the RNeasy mini kit (Qiagen, Hilden, Germany), treated with DNase I (Sigma), then reverse-transcribed with M-MLV reverse transcriptase (Promega, Foster, CA, USA). Quantitative PCR was performed on the CFX384 TouchTM Real-Time PCR Detection System (BioRad, Watford, Herts, UK). The gene expression was normalized using the geometric mean of three stable reference genes (Nono, Ywhaz and Hprt) or 18s. Sequences and details of all qPCR primers and assay probe sets are given in the Supplementary Table S1.

\subsection{Bone-Marrow-Derived Macrophages (BMDM)}

BMDM were isolated as described previously [19]. Cells were cultured and matured for seven days in DMEM supplemented with $10 \%$ Foetal Bovine Serum (FBS, ThermoFisher Scientific, Perth, UK), 20\% L929 conditioned media, $100 \mathrm{U} / \mathrm{mL}$ penicillin, $100 \mathrm{mg} / \mathrm{mL}$ Streptomycin (ThermoFisher Scientific, UK), $1 \mathrm{mM}$ sodium Pyruvate (Gibco, Grand Island, NY, USA), 1x MEM Non-essential Amino Acid (Sigma) and $0.25 \mathrm{mM} \beta$-mercaptoethanol (Sigma) in untreated Petri dishes. For the LPS treatment, BMDM were seeded in six-well tissue-culture plates and challenged with $100 \mathrm{ng} / \mathrm{mL}$ LPS for $4 \mathrm{~h}$.

\subsection{Bacterial Infection}

Staphylococcus aureus SH1000 mCherry was kindly provided by Professor Simon Foster, Krebs Institute, University of Sheffield, UK. Bacteria were added to macrophages in Hank's Balanced Salt Solution (Gibco) at a multiplicity of infection (MOI) of 5. At one hour post-infection, the cells were washed with PBS (Sigma-Aldrich, St. Louis, MO, USA) and incubated for $30 \mathrm{~min}$ in BMDM growth media supplemented with $100 \mu \mathrm{g} / \mathrm{mL}$ gentamicin (ThermoFisher Scientific, UK). The infected BMDM were maintained in $5 \mu \mathrm{g} / \mathrm{mL}$ gentamicin. At indicated times, cells were lysed in 0.1\% Triton X-100 (Sigma-Aldrich), and serial dilutions were plated onto $\mathrm{BHI}$ agar to determine colony-forming units (CFU).

\subsection{Immunofluorescence}

Infected BMDM plated on glass coverslips were fixed with $4 \%$ paraformaldehyde (PFA, Agar Scientific, Stansted, UK) and permeabilized in 0.2\% Triton X-100 (Sigma-Aldrich), $0.2 \%$ bovine serum albumin (BSA, Sigma-Aldrich) and $50 \mathrm{mM} \mathrm{NH} 4 \mathrm{Cl}$ (Sigma-Aldrich). Cells were incubated with anti-LAMP-1 antibody (\#1D4B, Developmental Studies Hybridoma Bank) and then Alexa Fluor ${ }^{\circledR} 488$ secondary antibody (Invitrogen, Carlsbad, CA, USA). Images were acquired on a PerkinElmer Spinning Disk confocal microscope linked to a Hamamatsu CMOS ORCA Flash 4.0 camera. An image analysis was performed using Volocity software.

\subsection{Data Analysis}

All data are presented as mean \pm SEM and were analyzed by an unpaired two-tailed Student's $t$ test or two-way analysis of variance with a Bonferroni post-hoc test, as appropriate, using GraphPad Prism. A p-value $<0.05$ was considered statistically significant.

\section{Results}

In order to examine the role of seipin deficiency in the macrophage function, we crossed $B s c l 2^{(\mathrm{fl} / \mathrm{fl})}$ mice [8] with $B s c l 2^{(\mathrm{fl} / \mathrm{wt})}$ mice expressing Cre recombinase controlled by the Lyz2 promoter (LysM-Cre) to generate myeloid-specific Bscl2 knockout (LysM-B2KO) mice (Figure 1A). The analysis of multiple tissues and bone-marrow-derived macrophages (BMDM) revealed that Bscl2 mRNA levels were readily detectable in BMDM from control mice and similar to the levels in gonadal white adipose tissue (gWAT, Figure 1B). Bscl2 mRNA expression was significantly reduced in BMDM from LysM-B2KO mice but was unaltered in other tested tissues, including liver, spleen and brown adipose tissue (BAT). A significant increase in the Bscl2 mRNA expression was however observed in gWAT (Figure 1B). The characterization of male and female myeloid-specific Bscl2 knock- 
out (LysM-B2KO) mice revealed no significant differences in body weight (Figure 1C,D), fat mass (Figure 1E,F) or lean mass (Figure S1A,B) when compared to littermate controls (CTRL). The glucose tolerance tests indicated that neither male nor female LysM-B2KO mice were glucose intolerant at 24 weeks of age (Figure 1G,H). To assess whether Bscl2 deficiency plays a role in the inflammatory response to Toll-like receptor 4 (TLR4) activation, LysM-B2KO mice were injected with a sub-lethal dose of lipopolysaccharide (LPS, $1 \mathrm{mg} / \mathrm{Kg}$ ). This led to a significant reduction in the body temperature in male but not female mice, with no genotype effect (Figure 1I,J). This sexually dimorphic response in body temperature in male versus female mice has been previously observed by others in other mouse models in adulthood [20]. As expected from previous studies [21], the LPS treatment caused a significant decrease in the blood glucose levels in male and female control mice (Figure 1K,L). This response was not altered by myeloid Bscl2 deficiency. Similarly, the glucose tolerance was not significantly changed in male or female LysM-B2KO mice when compared to the controls three hours following LPS injections (Figure S1C,D). The serum triglyceride levels were unaltered by LPS injection in both males and females and were equivalent in the control and LysM-B2KO mice (Figure S1E,F).
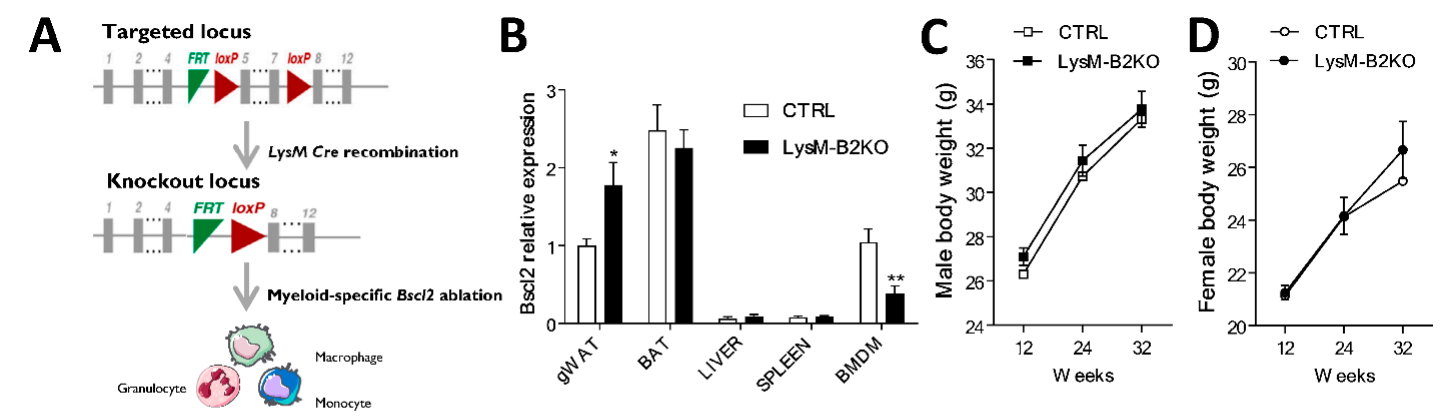

$\mathbf{E}$

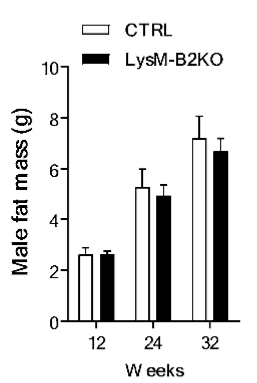

I

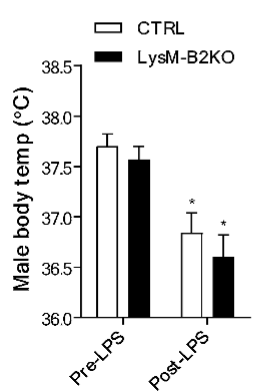

$\mathbf{F}$

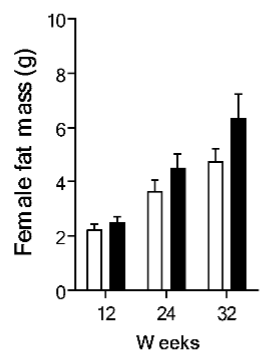

J

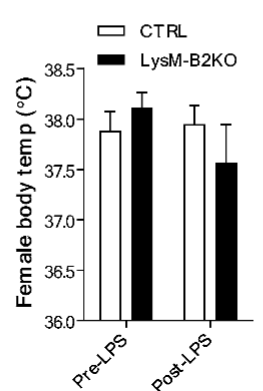

G

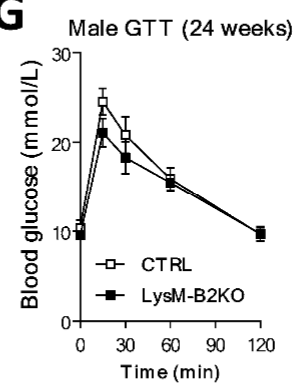

$\mathbf{K}$

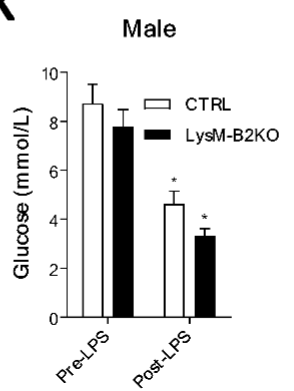

H

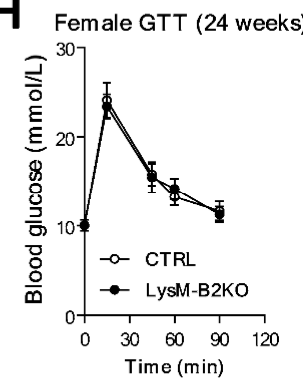

L

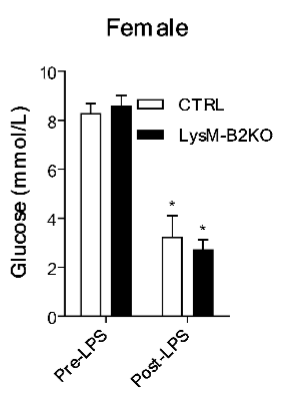

Figure 1. Physiological characteristics and lipopolysaccharide (LPS)-mediated inflammatory response in LysM-B2KO mice. (A) Targeting strategy for the conditional disruption of the Bscl2 gene in the myeloid lineage. (B) Bscl2 mRNA expression across different tissues and bone-marrow-derived macrophages (BMDM) of LysM-B2KO mice relative to the 18s gene. (C,D) Male and female body weight, $(\mathbf{E}, \mathbf{F})$ male and female fat mass, and $(\mathbf{G}, \mathbf{H})$ male and female glucose tolerance of LysM-B2KO mice at 24 weeks of age. Effect of LPS on the $(\mathbf{I}, \mathbf{J})$ body temperature and $(\mathbf{K}, \mathbf{L})$ serum glucose in male and female LysM-B2KO mice at 32 weeks of age (female $n=5-7$, male $n=7-10$ ). Data are represented as mean $\pm \mathrm{SEM},{ }^{*} p<0.05,{ }^{* *} p<0.01$. 
Next, we performed a serum analysis to determine any effects of seipin loss on the inflammatory response in LysM-B2KO mice. Serum insulin levels were not significantly altered in male or female LysM-B2KO mice when compared to control mice before or after LPS injection, and the quantitative insulin sensitivity check index (QUICKI) revealed that LysM-B2 mice were not insulin-resistant when compared to controls following the in vivo LPS challenge (Table 1). TLRs present on the surface of macrophages can sense LPS and trigger the synthesis of proinflammatory cytokines in order to eliminate the pathogen [22]. LysM-B2KO mice displayed decreased levels of serum TNF- $\alpha$ when compared to controls, although this was not significant (Table 1). IL-10 levels showed greater fluctuations among males and females following LPS injection, without being significant (Table 1). Taken together, our data indicate that LysM-B2KO mice responded to the in vivo LPS challenge in a similar manner as their littermate counterparts.

Table 1. Insulin, quantitative insulin sensitivity check index (QUICKI) and cytokine multiplex analysis of serum in LysMB2KO mice fasted for $5 \mathrm{~h}$ and subjected to $1 \mathrm{mg} / \mathrm{kg}$ LPS for $3 \mathrm{~h}$ (female $n=5-7$, male $n=7-10$ ). Data are represented as mean \pm SEM.

\begin{tabular}{cccccc}
\hline & & \multicolumn{2}{c}{ Male } & & Female \\
\cline { 2 - 6 } & Genotype & $\begin{array}{c}\text { Pre-LPS } \\
\text { Treatment }\end{array}$ & $\begin{array}{c}\text { Post-LPS } \\
\text { Treatment }\end{array}$ & $\begin{array}{c}\text { Pre-LPS } \\
\text { Treatment }\end{array}$ & $\begin{array}{c}\text { Post-LPS } \\
\text { Treatment }\end{array}$ \\
\hline \multirow{2}{*}{ Insulin $(\mu \mathrm{g} / \mathrm{L})$} & CTRL & $0.28 \pm 0.15$ & $1.75 \pm 1.51$ & $0.16 \pm 0.03$ & $0.21 \pm 0.07$ \\
& LysM-B2KO & $0.34 \pm 0.13$ & $0.99 \pm 0.46$ & $0.18 \pm 0.11$ & $0.39 \pm 0.08$ \\
QUICKI & CTRL & $0.34 \pm 0.03$ & $0.30 \pm 0.04$ & $0.36 \pm 0.02$ & $0.39 \pm 0.04$ \\
& LysM-B2KO & $0.33 \pm 0.02$ & $0.33 \pm 0.02$ & $0.37 \pm 0.03$ & $0.38 \pm 0.02$ \\
IL-10 (pg/mL) & CTRL & - & $453.91 \pm 104.65$ & - & $561.13 \pm 113.92$ \\
TNF- $\alpha(\mathbf{p g} / \mathbf{m L})$ & LysM-B2KO & - & $404.87 \pm 114.11$ & - & $865.17 \pm 408.16$ \\
& CTRL & - & $390.78 \pm 68.26$ & - & $394.17 \pm 387.63$ \\
& LysM-B2KO & - & $313.94 \pm 97.05$ & - & $200.21 \pm 107.88$ \\
\hline
\end{tabular}

To investigate further, BMDM were isolated from the male and female control and LysM-B2KO mice. As expected, male and female LysM-B2KO mice exhibited a significantly reduced Bscl2 expression, but no change in the expression of anti-inflammatory (Il-10) or proinflammatory cytokines (Tnfa, Il-6, Il-1b) was observed in the seipin-deficient cells (Figure 2A,B). BMDM from female control and LysM-B2KO mice were also examined in the absence or presence of stimulation with $100 \mathrm{ng} / \mathrm{mL}$ LPS for four hours. The induction of Il-10, Tnfa, Il-6, Il-1 $\beta$, iNos, Mcp1 was unchanged by the loss of seipin in LysM-B2KO BMDM, although the induction of $I l-1 \alpha$ was modestly but significantly greater (Figure $2 \mathrm{C}-\mathrm{I}$ ).

BMDM isolated from LysM-B2KO mice show significantly reduced but still clearly detectable levels of Bscl2 expression (Figure 2A,B). It is possible that this arose from an incomplete LysMCre-mediated recombination, and we were unable obtain western blots to accurately determine the resulting seipin protein levels. Therefore, to examine the effect of a complete Bscl2 deletion in this cell type, we next examined the innate immune response in macrophages isolated from global Bscl2 knockout (SKO) mice. These mice were generated by incubating fertilized $\mathrm{Bscl} 2^{(\mathrm{fl} / \mathrm{wt})}$ one-cell embryos with cell-permeable TAT-Cre recombinase in culture before reimplantation into surrogate dams (shown schematically in Figure $3 \mathrm{~A}$ ). These mice had the same gene deletion as the SKO mice we described previously [8] but were generated by this alternative method. Like other SKO mice, they had a similar body weight but a significantly reduced fat mass and increased lean mass compared to control mice (Figure S2A-E) along with elevated glycaemia in the fed state and fasting hyperinsulinaemia with a QUICKI analysis indicative of insulin resistance (Figure S2F-H). The Bscl 2 expression was decreased by more than $98 \%$ in SKO macrophages when compared to control samples (Figure 3B). However, similar to observations in LysM-B2KO BMDM, the basal and LPS-stimulated expression of anti- and proinflammatory cytokines was not significantly different between the control and SKO BMDM (Figure 3C-I). 
A

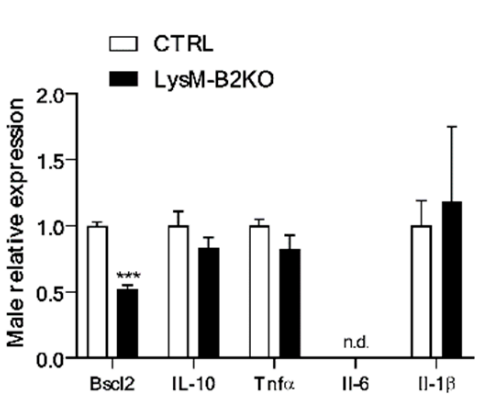

B

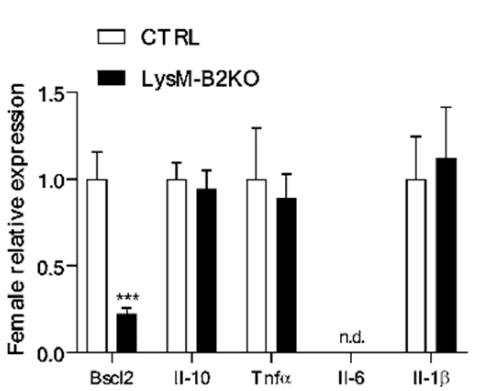

C

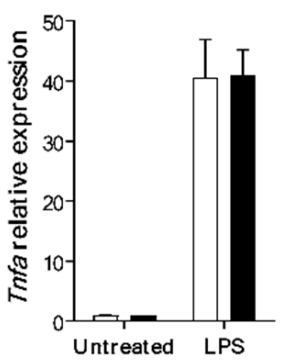

$\mathbf{F}$

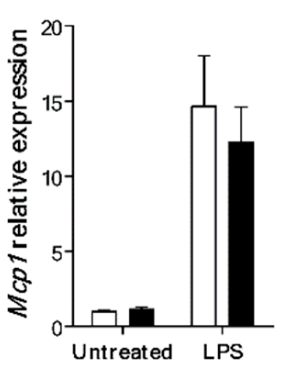

D

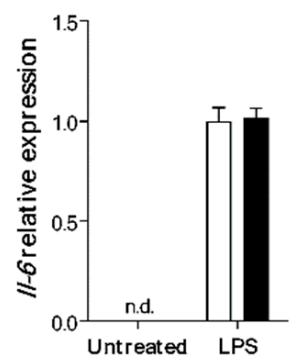

G

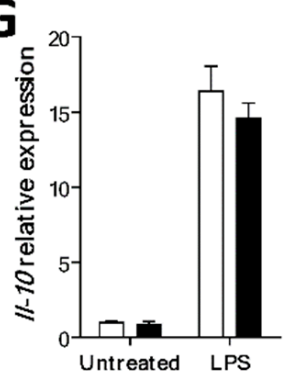

E

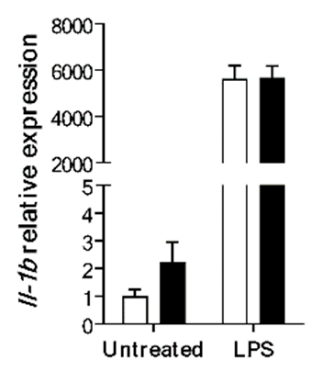

H

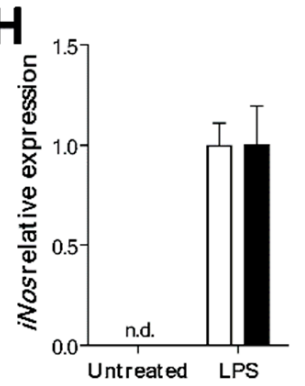

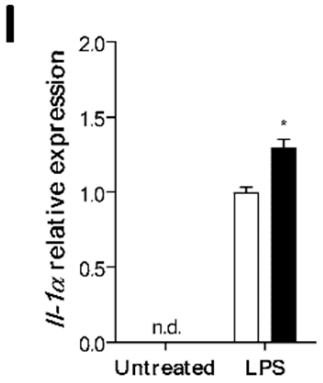

Figure 2. Lipopolysaccharides-mediated inflammatory responses in bone-marrow-derived macrophages. Basal mRNA levels of cytokines in BMDM isolated from (A) male and (B) female LysM-B2KO mice (female $n=6-7$, male $n=6-9$ ). (C-I) The treatment of female BMDM with LPS $(n=6)$ showed no significant difference in the expression of a panel of cytokines. Gene expression is normalized to three reference genes (NoNo, Ywhaz and Hprt). Data are represented as mean \pm SEM, ${ }^{*} p<0.05,{ }^{* * *} p<0.001$, n.d., not detectable.

Next, we examined the intracellular fate and the ability of SKO macrophages to phagocytose a common bacterial pathogen. Control and SKO BMDM were infected with Staphylococcus aureus SH1000 expressing mCherry. After $1.5 \mathrm{~h}$ post-infection, bacteria were internalized into macrophages and found in lysosome-associated membrane protein-1 (LAMP-1) positive vacuoles (Figure 3J). Sixty to seventy percent of intracellular S. aureus were found to colocalize with LAMP-1 vacuoles in both control and SKO macrophages (Figure 3K). To determine whether SKO macrophages could effectively kill S. aureus once internalized, bacterial survival was assessed using a gentamicin protection assay. We found that $S$. aureus clearance was similar in control and SKO BMDM, with less than 6\% of viable bacteria remaining $24 \mathrm{~h}$ after infection (Figure 3L,M). 
A
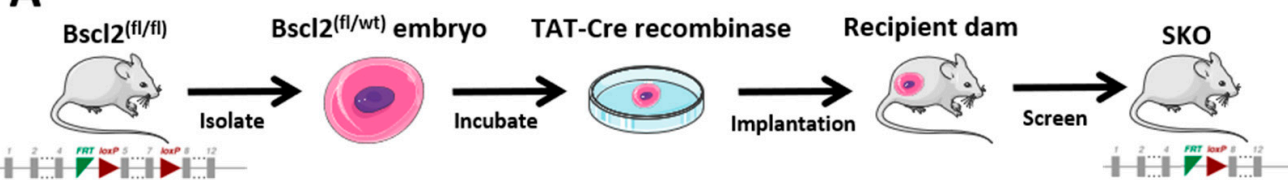

B

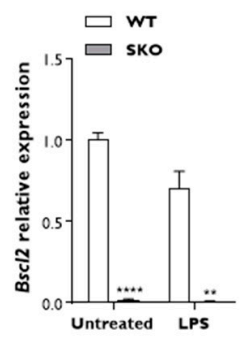

$\mathbf{F}$

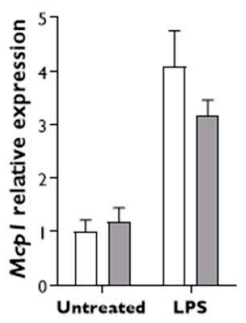

C

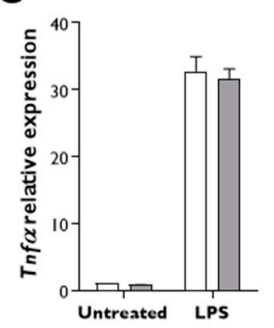

G

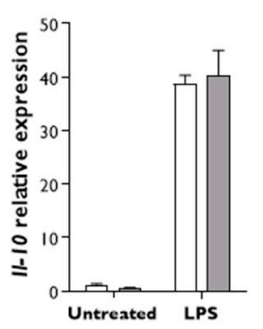

D

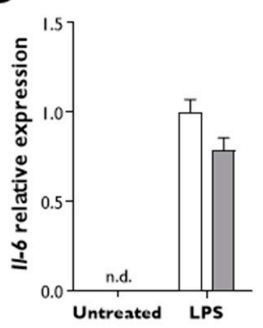

H

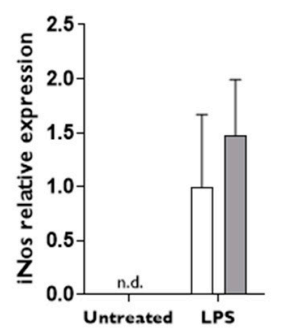

E

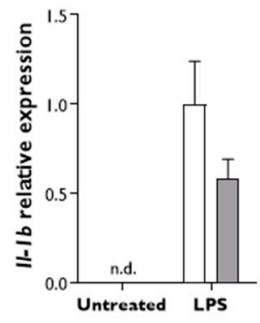

|

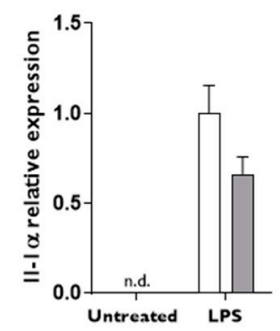

J

S. aureus

LAMP-I

Merged

K
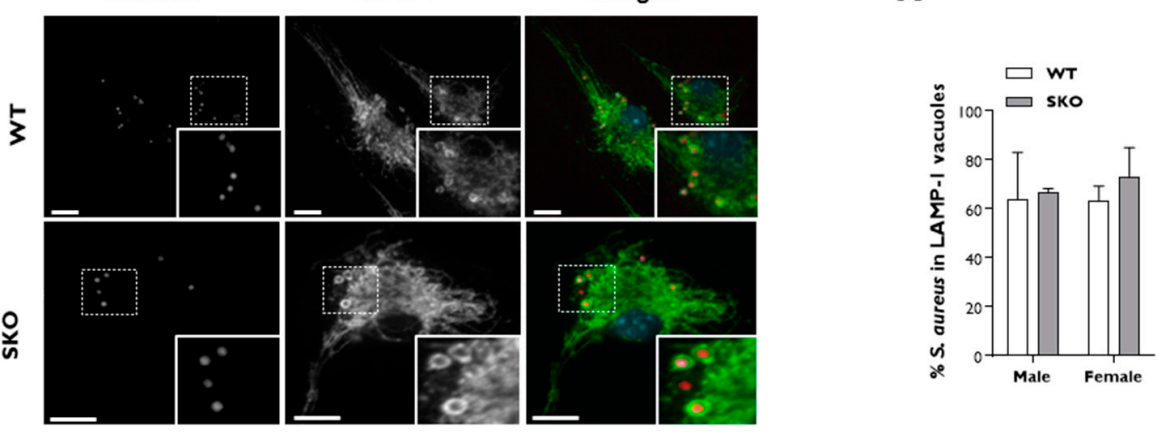

L

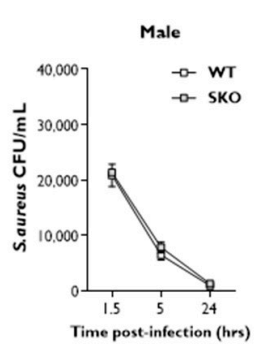

M

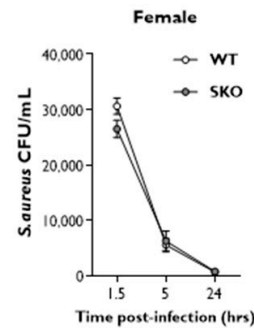

Figure 3. Bscl2 knockout (SKO) mice BMDM efficiently respond to lipopolysaccharides and Staphylococcus aureus challenge. (A) Strategy for the generation of SKO global knockout mice. qPCR was performed to evaluate the expression levels of (B) Bscl2 and (C-I) a panel of cytokines in female SKO BMDM challenged with LPS $(n=3)$. The gene expression was normalized to three reference genes (NoNo, Ywhaz and Hprt). Data are represented as mean \pm SEM, ${ }^{* *} p<0.01,{ }^{* * *} p<0.0001$, n.d., not detectable. (J) Representative confocal micrographs of the LAMP-1 distribution in BMDM infected with S. aureusmCherry $(n=6)$. Dotted boxes indicate selected regions magnified in the white boxes. Bars denote $14 \mu \mathrm{m}$. (K) Quantification of the LAMP-1 association with S. aureus-mCherry. Data are represented as mean \pm SEM. (L,M) Colony-forming units (CFU) of $S$. aureus inside male and female BMDM. 


\section{Discussion}

BSCL2 gene mutations cause severe lipodystrophy, in which liver disease and infection are the main causes of death [13]. Liver disease can be linked to the severe hepatic steatosis in these patients [1]. It is less clear why infections are linked to BSCL2 deficiency, but a direct effect in macrophages is credible. Seipin plays an important, evolutionarily conserved role in the biogenesis of lipid droplets [23], organelles that have been shown to regulate macrophage function and infection resolution, as has lipid metabolism [16,24]. In addition, $B S C L 2$ /seipin deficiency has been shown to induce ER stress [23], a process that has also been implicated in altered innate immunity and myeloid cell dysfunction in type 2 diabetes and atherosclerosis.

The findings presented here for the first time directly examined the consequence of Bscl2 deficiency in myeloid cells. We found no impairment of the innate immune response in LysM-B2KO mice challenged with endotoxin. Moreover, macrophages isolated from LysM-B2KO or fully seipin-deficient SKO mice displayed no alteration in LPS-induced antior proinflammatory cytokine responses. Additionally, SKO macrophages were capable of pathogen recognition, engulfment, phagolysosome maturation and clearance when infected with Staphylococcus aureus. Whilst we observed no dramatic changes in macrophage function in this study, we examined only LPS-induced immune responses. This is a rather artificial surrogate for bacterial infection in vivo, and it remains possible that a different result may be observed with more clinically relevant and accurate models of sepsis. Nonetheless, we believe that our findings provide substantial evidence that Bscl2 deficiency within macrophages does not directly impair the innate immune response. Therefore, it is unlikely that BSCL2 deficiency in the myeloid lineage per se significantly contributes to an increased risk of infection in CGL2 patients.

In light of our findings, it would appear that any increased risk of death from infection in CGL2 is likely to result as being secondary to adipose tissue deficiency and the severe metabolic disease observed in this condition. For example, it has been observed that patients with congenital leptin deficiency have impaired immunity and increased rates of death from infections [25]. Leptin is now known to be a key regulator of the innate and adaptive immune responses, with leptin deficiency or resistance leading to the dysregulation of inflammatory responses and increased susceptibility of infectious disease (Reviewed in [26]). Patients with CGL2 have a near complete loss of metabolic and mechanical adipose tissues. Consequently, this results in significant decreases in the circulating levels of the adipose-secreted hormone leptin. Additionally, both hyperglycaemia and dyslipidaemia have been implicated in the impairment of the normal innate immune response and in macrophage dysfunction in type 2 diabetes [27]. Of note, SKO mice are hyperglycaemic but do not display the hypertriglyceridemia observed in BSCL2-deficient patients [28]. However, this can be modeled more accurately in SKO mice crossed to a dyslipidemic ApoE-null background [29]. Thus, a comparison of the response to infection in SKO versus $\mathrm{SKO} / \mathrm{ApoE}-\mathrm{null}$ mice may permit a dissection of the effects driven by hyperglycaemia and hyperlipidemia. It is possible to speculate about other reasons that may place CGL2 patients at an increased risk of infection. Seipin is highly expressed in the central nervous system, and there could be centrally driven effects on the immune function that suppress the immune function of these patients. Overall, a better understanding of the mechanisms involved in this phenomenon may establish suitable conditions to examine therapeutic interventions that could decrease the susceptibility to infection and the mortality rate amongst patients with CGL2.

Supplementary Materials: The following are available online at https:/ /www.mdpi.com/2077-038 3/10/3/441/s1, Figures S1, S2 and Table S1.

Author Contributions: A.R. and G.D.M. designed, performed, analyzed the experiments and drafted the initial manuscript. A.B. performed the experiments. W.H., M.D., M.B. and P.N. designed and interpreted experiments. J.J.R. conceived the study, designed and interpreted the experiments. All authors have read and agreed to the published version of the manuscript. 
Funding: Work was supported by Diabetes UK (JJR;18/0005884, MD;17/0005621) the Medical Research Council (JJR; MR/L002620/1, MC/PC/15077), the British Heart Foundation (MD; PG/14/43/ 30889), The Agency for Science, Technology and Research, Singapore (A*STAR) (WH), The Wellcome Trust (ISSF Funding to GDM) and the European Union's Horizon 2020 ERC consolidator award (MB:2016-726152-TYPHI).

Institutional Review Board Statement: In vivo experiments were approved by the University of Aberdeen Ethics Review Board and performed under project license P94B395E0, approved by the UK Home Office under the Animals Scientific Procedures Act 1986.

Informed Consent Statement: Not Applicable.

Data Availability Statement: Data are available on request from the corresponding author.

Acknowledgments: A.R., J.J.R. and P.N. thank Curtin University and Aberdeen University for the collaborative PhD scholarship awarded to A.R. The authors would like to thank Simon Foster (Krebs Institute, University of Sheffield, UK) for kindly providing the Staphylococcus aureus SH1000 mCherry strain.

Conflicts of Interest: The authors declare no conflict of interest. The funders had no role in the design of the study; in the collection, analyses, or interpretation of data; in the writing of the manuscript; or in the decision to publish the results.

\section{References}

1. Garg, A. Lipodystrophies: Genetic and acquired body fat disorders. J. Clin. Endocrinol. Metab. 2011, 96, 3313-3325. [CrossRef]

2. Magre, J.; Delepine, M.; Khallouf, E.; Gedde-Dahl, T., Jr.; Van Maldergem, L.; Sobel, E.; Papp, J.; Meier, M.; Megarbane, A.; Bachy, A.; et al. Identification of the gene altered in Berardinelli-Seip congenital lipodystrophy on chromosome 11q13. Nat. Genet. 2001, 28, 365-370. [CrossRef] [PubMed]

3. Payne, V.A.; Grimsey, N.; Tuthill, A.; Virtue, S.; Gray, S.L.; Dalla Nora, E.; Semple, R.K.; O'Rahilly, S.; Rochford, J.J. The human lipodystrophy gene BSCL2/seipin may be essential for normal adipocyte differentiation. Diabetes 2008, 57, 2055-2060. [CrossRef] [PubMed]

4. Chen, W.; Yechoor, V.K.; Chang, B.H.; Li, M.V.; March, K.L.; Chan, L. The human lipodystrophy gene product Berardinelli-Seip congenital lipodystrophy 2/seipin plays a key role in adipocyte differentiation. Endocrinology 2009, 150, 4552-4561. [CrossRef] [PubMed]

5. Cui, X.; Wang, Y.; Tang, Y.; Liu, Y.; Zhao, L.; Deng, J.; Xu, G.; Peng, X.; Ju, S.; Liu, G.; et al. Seipin ablation in mice results in severe generalized lipodystrophy. Hum. Mol. Genet. 2011, 20, 3022-3030. [CrossRef] [PubMed]

6. Chen, W.; Chang, B.; Saha, P.; Hartig, S.M.; Li, L.; Reddy, V.T.; Yang, Y.; Yechoor, V.; Mancini, M.A.; Chan, L. Berardinelli-seip congenital lipodystrophy 2 /seipin is a cell-autonomous regulator of lipolysis essential for adipocyte differentiation. Mol. Cell Biol. 2012, 32, 1099-1111. [CrossRef] [PubMed]

7. Prieur, X.; Dollet, L.; Takahashi, M.; Nemani, M.; Pillot, B.; Le May, C.; Mounier, C.; Takigawa-Imamura, H.; Zelenika, D.; Matsuda, F.; et al. Thiazolidinediones partially reverse the metabolic disturbances observed in Bscl2/seipin-deficient mice. Diabetologia 2013, 56, 1813-1825. [CrossRef]

8. Mcilroy, G.D.; Suchacki, K.; Roelofs, A.J.; Yang, W.; Fu, Y.; Bai, B.; Wallace, R.J.; De Bari, C.; Cawthorn, W.P.; Han, W.; et al. Adipose specific disruption of seipin causes early-onset generalised lipodystrophy and altered fuel utilisation without severe metabolic disease. Mol. Metab. 2018, 10, 55-65. [CrossRef] [PubMed]

9. Sim, M.F.; Dennis, R.J.; Aubry, E.M.; Ramanathan, N.; Sembongi, H.; Saudek, V.; Ito, D.; O’Rahilly, S.; Siniossoglou, S.; Rochford, J.J. The human lipodystrophy protein seipin is an ER membrane adaptor for the adipogenic PA phosphatase lipin 1. Mol. Metab. 2012, 2, 38-46. [CrossRef]

10. Talukder, M.M.; Sim, M.F.; O’Rahilly, S.; Edwardson, J.M.; Rochford, J.J. Seipin oligomers can interact directly with AGPAT2 and lipin 1, physically scaffolding critical regulators of adipogenesis. Mol. Metab. 2015, 4, 199-209. [CrossRef]

11. Castro, I.G.; Eisenberg-Bord, M.; Persiani, E.; Rochford, J.J.; Schuldiner, M.; Bohnert, M. Promethin Is a Conserved Seipin Partner Protein. Cells 2019, 8, 268. [CrossRef] [PubMed]

12. Sim, M.F.M.; Persiani, E.; Talukder, M.M.U.; Mcilroy, G.D.; Roumane, A.; Edwardson, J.M.; Rochford, J.J. Oligomers of the lipodystrophy protein seipin may co-ordinate GPAT3 and AGPAT2 enzymes to facilitate adipocyte differentiation. Sci. Rep. 2020, 10, 3259. [CrossRef]

13. Lima, J.G.; Nobrega, L.H.C.; Lima, N.N.; Dos Santos, M.C.F.; Silva, P.H.D.; Baracho, M.F.P.; Lima, D.N.; de Melo Campos, J.T.A.; Ferreira, L.C.; Freire Neto, F.P.; et al. Causes of death in patients with Berardinelli-Seip congenital generalized lipodystrophy. PLoS ONE 2018, 13, e0199052. [CrossRef] [PubMed]

14. Bohnert, M. New friends for seipin-Implications of seipin partner proteins in the life cycle of lipid droplets. Semin. Cell Dev. Biol. 2020, 108, 24-32. [CrossRef] [PubMed] 
15. Dichlberger, A.; Kovanen, P.T.; Schneider, W.J. Mast cells: From lipid droplets to lipid mediators. Clin. Sci. 2013, 125, 121-130. [CrossRef]

16. Melo, R.C.; Dvorak, A.M. Lipid body-phagosome interaction in macrophages during infectious diseases: Host defense or pathogen survival strategy? PLoS Pathog. 2012, 8, e1002729. [CrossRef]

17. Kim, K.; Kim, H.; Lee, D. Site-specific modification of genome with cell-permeable Cre fusion protein in preimplantation mouse embryo. Biochem. Biophys. Res. Commun. 2009, 388, 122-126. [CrossRef]

18. Katz, A.; Nambi, S.S.; Mather, K.; Baron, A.D.; Follmann, D.A.; Sullivan, G.; Quon, M.J. Quantitative insulin sensitivity check index: A simple, accurate method for assessing insulin sensitivity in humans. J. Clin. Endocrinol. Metab. 2000, 85, 2402-2410. [CrossRef]

19. Owen, C.; Czopek, A.; Agouni, A.; Grant, L.; Judson, R.; Lees, E.K.; Mcilroy, G.D.; Goransson, O.; Welch, A.; Bence, K.K.; et al. Adipocyte-specific protein tyrosine phosphatase $1 \mathrm{~B}$ deletion increases lipogenesis, adipocyte cell size and is a minor regulator of glucose homeostasis. PLoS ONE 2012, 7, e32700. [CrossRef]

20. Cai, K.C.; van Mil, S.; Murray, E.; Mallet, J.F.; Matar, C.; Ismail, N. Age and sex differences in immune response following LPS treatment in mice. Brain Behav. Immun. 2016, 58, 327-337. [CrossRef]

21. Raetzsch, C.F.; Brooks, N.L.; Alderman, J.M.; Moore, K.S.; Hosick, P.A.; Klebanov, S.; Akira, S.; Bear, J.E.; Baldwin, A.S.; Mackman, N.; et al. Lipopolysaccharide inhibition of glucose production through the Toll-like receptor-4, myeloid differentiation factor 88, and nuclear factor kappa b pathway. Hepatology 2009, 50, 592-600. [CrossRef] [PubMed]

22. Ciesielska, A.; Matyjek, M.; Kwiatkowska, K. TLR4 and CD14 trafficking and its influence on LPS-induced pro-inflammatory signaling. Cell Mol. Life Sci. 2020. [CrossRef] [PubMed]

23. Craveiro Sarmento, A.S.; de Azevedo Medeiros, L.B.; Agnez-Lima, L.F.; Lima, J.G.; de Melo Campos, J.T.A. Exploring Seipin: From Biochemistry to Bioinformatics Predictions. Int. J. Cell Biol. 2018, 2018, 5207608. [CrossRef]

24. Remmerie, A.; Scott, C.L. Macrophages and lipid metabolism. Cell Immunol. 2018, 330, 27-42. [CrossRef] [PubMed]

25. Ozata, M.; Ozdemir, I.C.; Licinio, J. Human leptin deficiency caused by a missense mutation: Multiple endocrine defects, decreased sympathetic tone, and immune system dysfunction indicate new targets for leptin action, greater central than peripheral resistance to the effects of leptin, and spontaneous correction of leptin-mediated defects. J. Clin. Endocrinol. Metab. 1999, 84, 3686-3695. [PubMed]

26. Maurya, R.; Bhattacharya, P.; Dey, R.; Nakhasi, H.L. Leptin Functions in Infectious Diseases. Front. Immunol. 2018,9 , 2741. [CrossRef]

27. Ratter, J.M.; Tack, C.J.; Netea, M.G.; Stienstra, R. Environmental Signals Influencing Myeloid Cell Metabolism and Function in Diabetes. Trends Endocrinol. Metab. 2018, 29, 468-480. [CrossRef]

28. Dollet, L.; Magre, J.; Cariou, B.; Prieur, X. Function of seipin: New insights from Bscl2/seipin knockout mouse models. Biochimie 2014, 96, 166-172. [CrossRef]

29. Liao, J.; Liu, X.; Gao, M.; Wang, M.; Wang, Y.; Wang, F.; Huang, W.; Liu, G. Dyslipidemia, steatohepatitis and atherogenesis in lipodystrophic apoE deficient mice with Seipin deletion. Gene 2018, 648, 82-88. [CrossRef] 